\title{
Splicing variants of ADAR2 and ADAR2-mediated RNA editing in glioma (Review)
}

\author{
YAO FU, XINGLI ZHAO, ZHAOHUI LI, JUN WEI and YU TIAN \\ Department of Neurosurgery, China-Japan Union Hospital, Jilin University, \\ Changchun, Jilin 130033, P.R. China
}

Received May 17, 2015; Accepted May 26, 2016

DOI: $10.3892 / \mathrm{ol} .2016 .4734$

\begin{abstract}
The roles of alternative splicing and RNA editing in gene regulation and transcriptome diversity are well documented. Adenosine deaminases acting on RNA (ADARs) are responsible for adenosine-to-inosine (A-to-I) editing and exemplify the complex association between RNA editing and alternative splicing. The self-editing activity of ADAR2, which acts on its own pre-mRNA, leads to its alternative splicing. Alternative splicing occurs independently at nine splicing sites on ADAR2 pre-mRNA, generating numerous alternative splicing variants with various catalytic activities. A-to-I RNA editing is important in a range of physiological processes in humans and is associated with several diseases, including amyotrophic lateral sclerosis, mood disorders, epilepsy and glioma. Reduced editing at the glutamine/arginine site of the AMPA receptor subunit GluA2 in glioma, without any alteration in ADAR2 expression, is a notable phenomenon. Several studies have tried to explain this alteration in the catalytic activity of ADAR2; however, the underlying mechanism remains unclear. The present review summarizes the relevant literature and shares experimental results concerning ADAR2 alternative splicing. In particular, the present review demonstrates that shifts in the relative abundance of the active and inactive splicing variants of ADAR2 may reduce the ADAR2 editing activity in glioma. Dominant expression of ADAR2 splicing variant with low enzyme activity causes reduced RNA editing of GluA2 subunit at the glutamine/arginine site in glioma.
\end{abstract}

Correspondence to: Professor $\mathrm{Yu}$ Tian, Department of Neurosurgery, China-Japan Union Hospital, Jilin University, 126 Xiantai Street, Changchun, Jilin 130033, P.R. China

E-mail: tianyu@jlu.edu.cn

Abbreviations: A-to-I, adenosine-to-inosine; ADAR, adenosine deaminase acting on RNA; ASV, alternative splicing variant; dsRBD, dsRNA-binding domain

Key words: alternative splicing, splicing variant, ADAR2, RNA editing, glioma

\section{Contents}

1. Introduction

2. RNA editing and ADARs

3. Alternative splicing

4. Alternative splicing of human ADAR2 mRNA

5. Reduced RNA editing in glioma

6. ADAR2 ASVs regulate RNA editing in glioma

7. Conclusion

\section{Introduction}

Carcinogenesis is a complex, multistage process. Advances in gene sequencing technology have demonstrated that certain DNA mutations and chromosomal abnormalities are important in tumorigenesis and tumor development. Aberrations in RNA, a central element in gene expression, are also vital in tumorigenesis, tumor development and malignant growth at the posttranscriptional and epigenetic level. Posttranscriptional modifications, including RNA editing and alternative splicing, render it possible to diversify the transcriptome, while restricting the size of the genome. Members of the adenosine deaminase acting on RNA (ADAR) family of enzymes, which catalyze adenosine-to-inosine (A-to-I) RNA editing, have been associated with alternative splicing in glioma $(1,2)$. Levels of A-to-I RNA editing are reportedly reduced in glioma, and alternative splicing variants (ASVs) of ADAR2 are expressed at various levels (3). Notably, the expression of ADAR2 mRNA is unaltered in glioma, and the underlying mechanism of this phenomenon remains unclear. The present review discusses alternative splicing and RNA editing in glioma, specifically in terms of the ADAR2 spliced isoforms.

\section{RNA editing and ADARs}

RNA editing was first identified in trypanosomes by Benne et al (4), who concluded that alterations in nucleotide sequences occur during or following transcription of the frameshift gene by a RNA editing process. A subsequent study identified a RNA duplex unwinding activity when antisense RNA was injected into fertilized frog eggs (5). The unwinding activity was later demonstrated to arise from structural alterations in the RNA when adenosine (A) is converted 
to inosine (I) (6). The following types of RNA editing have been identified (7): Uridine (U) insertion or deletion (8); cytosine (C) insertion and dinucleotide insertion (9); small nucleolar RNA-mediated nucleotide modification of ribosomal RNAs (10); transfer (t)RNA editing (11); C-to-U editing (12); and A-to-I editing (13). In mammals, A-to-I editing is the most common type of RNA editing causing genetic diversity (14). A-to-I RNA editing occurs at over one hundred million genomic sites, which are located in the majority of human genes (15). ADAR enzymes, responsible for the catalytic conversion of A to I (14), share a highly conserved catalytic deaminase domain (DM) at their C-terminal, which binds to double-stranded DNA (dsRNA) synergistically with the $\mathrm{N}$-terminus dsRNA-binding domain (dsRBD) (16-18). In vertebrates, three members of the ADAR family (ADAR1, ADAR2 and ADAR3) have been identified (19). There are three dsRBDs for ADAR1, and two for ADAR2 and ADAR3 (18). ADAR1 and ADAR2 are ubiquitously expressed in humans and exhibit catalytic activity, whereas ADAR3 is expressed specifically in the brain and has no catalytic activity (14). ADAR3 has been revealed to competitively inhibit deaminase activities of other ADARs by binding to dsRNA (20). The adenosine deaminase reaction catalyzed by ADAR2 is site-specific (21), as demonstrated in studies of the GluA2 subunit of glutamate AMPA receptor (21-23), in which the conversion of a glutamine (Q) to an arginine ( $R$ ) codon is exclusively mediated by ADAR2 (23). ADAR2 activity is essential for brain development and function (24), and $>99.9 \%$ of RNA editing occurs at the Q/R site of GluA2 in the human central nervous system (25). In addition, RNA editing plays a role in controlling microRNA (miRNA) biogenesis (26). A recent study of the mouse brain revealed that reproducible alterations in the sequence and abundance of mature miRNAs are induced by ADAR2 (27). Furthermore, ADAR2-mediated editing is site-specific, as opposed to sequence-specific, and ADAR 2 edits the coding and noncoding regions of mRNAs $(28,29)$. Therefore, a single-base modification during the recoding process during editing may affect the coding potential of the RNA and its splicing.

\section{Alternative splicing}

Studies conducted in 1977 revealed that the coding regions in DNA sequences are not continuous, and the final tRNA or mRNA is a spliced product (30,31). In 1978, Gilbert presented 'Why genes in pieces?' (32), in which the terms 'intron' and 'exon' were first introduced. The research by Gilbert led to an additional study, which confirmed that alternative splicing occurs in eukaryotic cells (33). In 1994, the level of alternatively spliced human genes was estimated to be only $5 \%$ (34). However, by the 21 st century, data from The International Genome Sequencing Consortium predicted that $\geq 50 \%$ of human genes are alternatively spliced $(33,35,36)$. In 2008, Wang et al (37) and Pan et al (38) demonstrated that $>90 \%$ of multi-exon genes are alternatively spliced, and the majority of these are specifically alternatively spliced tissue. Wang et al (37) also described various types of alternative splicing, including exon skipping, intron retention, alternative $5^{\prime}$ splice sites, alternative 3 ' splice sites, mutually exclusive exons, mutually exclusive $5^{\prime}$ untranslated regions (UTRs), mutually exclusive 3 ' UTRs and tandem UTRs. Alternative splicing results in the expression of diverse proteins and affects transcription factors, cell signaling, transmembrane proteins and secreted extracellular proteins (39). Consequently, the structural and functional alterations in these proteins and signaling pathways may be involved in carcinogenesis (39).

\section{Alternative splicing of human ADAR2 mRNA}

The human ADAR2 gene is located on the long arm of chromosome 21 (21q22.3) and spans $~ 153 \mathrm{kbp}$ (40-42). A study by Slavov and Gardiner (42) revealed that the genomic structure of the human ADAR2 gene consists of 15 exons. The structure of ADAR2 mRNA is illustrated in Fig. 1. Kawahara et al (43) followed the exon and intron identifiers reported by Slavov and Gardiner to divide ADAR2 mRNA into four regions based on alternative splicing sites: The first region includes exons $2-1$, in which two ASVs occur; the second region includes exons 2-3, in which three ASVs occur; the third region includes exons 4-8, in which two ASVs occur; and the fourth region includes exons 9-10. So far, a total of nine splicing sites in ADAR2 mRNA have been confirmed (42-48). Alternative splicing at these sites occurs independently, resulting in dozens of ADAR2 spliced isoforms. This renders it challenging to analyze tissue-specific and developmental-stage-dependent properties of the ADAR2 ASVs in vivo (49). Studies have identified that alternative splicing occurs at exon 2 and exon 4-6, which are dsRBD and DM coding regions, and these affect the enzyme activity of ADAR2 $(43,44,46)$. In addition, inclusion of exon 5 a results in the generation of a protein, which has $\sim 50 \%$ reduction in activity (44). The exception is the splicing variants, which have a distinctive truncated shorter $\mathrm{C}$-terminal structure, and exhibit no editing activity, if splicing occurs at exon 9 (45). Agranat et al (48) described a splicing event that the 93 nucleotide sequence located in intron 7 was included as exon 7a. This also occurs outside ADAR2 function domain coding regions, but does not lead to a catalytic activity product. Alternative exon $7 \mathrm{a}$ is expressed tissue-specifically, with high levels in the skeletal muscle, heart and testis, and low levels in the brain (48). Alternative splicing sites in human ADAR2 mRNA are summarized in Table I, which is adapted from a previous study by the present authors (50).

\section{Reduced RNA editing in glioma}

Although extensive sequencing and analysis of the human genome have revealed a clear association between genes and cancer, numerous questions have also been raised. Currently, in the postgenomic era, the field of epigenetics has received much attention. Epigenetic alterations, including alternative splicing and ADAR-mediated A-to-I RNA editing, have been associated with several types of cancer, including breast cancer, neuroblastoma and hepatocellular carcinoma, and there is a particularly clear association with glioma $(3,39,51)$.

Glioma is the most common type of tumor in the central nervous system, and is classified into four grades that reflect the degree of malignancy. Glioblastoma multiforme (grade IV) is the most aggressive type of glioma and is fatal (1). An association between RNA editing and glioma, particularly the pathogenesis of glioblastoma, was first identified by Maas et al (1), who demonstrated that there was 
Table I. Summary of alternative splicing sites in human ADAR2 mRNA.

Effect on

\begin{tabular}{|c|c|c|c|c|c|}
\hline Author, year & ASV, exon & Effect on ADAR2 transcript & Effect on ADAR2 protein & catalytic activity & (Ref.) \\
\hline Slavov and Gardiner, 2002 & $-1-1$ & Inclusion of exon 1a & $\begin{array}{l}\text { 28-amino-acid N-terminal } \\
\text { extension }\end{array}$ & Unknown & (42) \\
\hline Kawahara et al, 2005 & $1-3$ & Skipping of exon 2 & $\begin{array}{l}\text { Generation of a 12-amino-acid } \\
\text { protein }\end{array}$ & None & (43) \\
\hline Kawahara et al, 2005 & $9-10$ & Inclusion of intron 9 & Unknown & Unknown & (43) \\
\hline Kawahara et al, 2005 & 9 & $\begin{array}{l}\text { Splices exon } 9,83 \mathrm{nt} \\
\text { downstream from stop codon }\end{array}$ & Unknown & Unknown & (43) \\
\hline Gerber et al, 1997 & $5-6$ & Inclusion of exon $5 \mathrm{a}$ & $\begin{array}{l}\text { Insertion of AluJ cassette in } \\
\text { the catalytic domain }\end{array}$ & Decreased & (44) \\
\hline Lai et al, 1997 & 9 & $\begin{array}{l}\text { Truncates } 3^{\prime} \text { end of the coding } \\
\text { region }\end{array}$ & $\begin{array}{l}\text { Replacement of } 29 \text { C-terminal } \\
\text { residues with } 2 \text { amino acids }\end{array}$ & None & $(45)$ \\
\hline Rueter et al, 1999 & $1-2$ & $\begin{array}{l}\text { Addition of } 47 \text { nt to } 5^{\prime} \text { end of } \\
\text { exon } 2\end{array}$ & Generation of a $9-\mathrm{kDa}$ protein & Decreased & (46) \\
\hline Maas and Gommans, 2009 & $-1-1$ & Inclusion of exon 0 & $\begin{array}{l}\text { 49-amino-acid N-terminal } \\
\text { extension }\end{array}$ & Unknown & (47) \\
\hline Agranat et al, 2010 & $7-8$ & Inclusion of exon $7 \mathrm{a}$ & $\begin{array}{l}\text { Nonsense-mediated mRNA } \\
\text { decay }\end{array}$ & None & $(48)$ \\
\hline
\end{tabular}

Adapted from Li et al (3). ADAR, adenosine deaminases acting on RNA; ASV, alternative splicing site; nt, nucleotide.

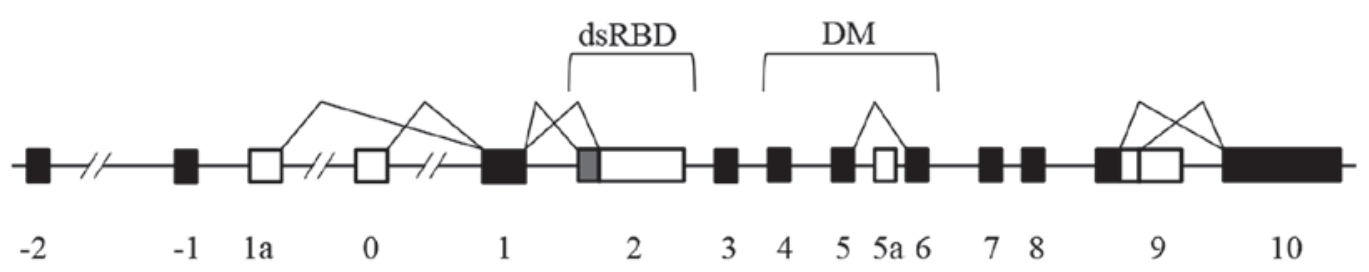

Figure 1. Exon-intron structure of human ADAR2 gene. Boxes illustrate exons, while lines illustrate introns. Filled boxes indicates coding and open boxes indicate non-coding. Shaded part of exon 2 indicates the 47 nucleotide cassette, where self-editing occurs, leading to the formation of a splicing site. ADAR, adenosine deaminases acting on RNA; dsRBD, N-terminus dsRNA-binding domain; DM, deaminase domain.

reduced editing at the $\mathrm{Q} / \mathrm{R}$ site of GluR-B, with no corresponding alteration in ADAR2 expression and no difference in the alternative splicing of ADAR2 mRNA in tumor and normal tissues. Cellular mechanisms that regulate ADAR2 catalytic activity are unknown, but may involve posttranslational modification or controlled subcellular localization of ADAR2 (1). Another study demonstrated that a reduction in editing levels was associated with the grade of malignancy in pediatric astrocytomas, which was attributed to altered ADAR2 catalytic activity (2). Furthermore, that study reported that ADAR2 overexpression inhibited cell proliferation and migration in vitro. In addition, alternative splicing within exon 2 of the ADAR1 pre-mRNA in high-grade tumors generated a $110-\mathrm{kDa}$ protein, as opposed to the full-length 150-kDa protein. Overexpressed ASVs of ADAR1 are hypothesized to form heterodimers with ADAR2, disrupting the balance between ADAR1, ADAR2 and ADAR3, and competing for specific ADAR2 editing activity at the $\mathrm{Q} / \mathrm{R}$ site of GluR-B (2). These findings suggest that the alternative splicing events in ADAR1 regulate ADAR2-mediated RNA editing.
A previous study demonstrated a significant loss of ADAR2 editing activity in newly diagnosed and recurrent pediatric high-grade astrocytoma (52). Notably, ADAR2 editing activity was substantially rescued in the only patient with prolonged survival, suggesting that ADAR2 activity/expression is a possible prognostic marker. Other results suggest attenuated A-to-I editing of miRNA-376a* promotes invasiveness of glioblastoma cells in vitro and orthotopic xenograft mouse models (53). In addition, a recent study demonstrated that ADAR2 editing activity inhibits glioblastoma growth by modulating the cell division cycle $14 \mathrm{~B} / \mathrm{S}$-phase kinase-associated protein 2/p21/p27 axis (54).

\section{ADAR2 ASVs regulate RNA editing in glioma}

Reduced RNA editing without a significant alteration in ADAR2 expression has been widely documented (1-3,55); however, the underlying mechanism remains unclear. Due to the large number of ADAR2 ASVs, ADAR2 expression may not associate fully with its editing activity. This raises the 
question of whether alternative splicing is another regulatory factor that may interfere with ADAR2-mediated RNA editing. However, the precise association between RNA editing and alternative splicing is complex and remains unclear.

Rueter et al (46) demonstrated that the addition of 47 nucleotides to the $5^{\prime}$ end of exon 2 occurs subsequent to RNA editing within intron 1 , which reduces ADAR2 activity in vivo. A previous study by the present authors revealed the expression levels of this ADAR2 ASV in human glioma tissues and glioma-derived U251 and BT325 cell lines (55); the ADAR2 ASV was expressed in $10 \%$ of low-grade astrocytomas, $16.7 \%$ of oligodendrogliomas, $12.5 \%$ of anaplastic astrocytomas and $25 \%$ of glioblastomas multiforme. In addition, the increased expression of this self-editing-induced ASV corresponded to the increasing malignancy of the glioma, and its expression appeared to be associated with the malignant features of glioma, as identified in the glioblastoma multiforme group of ADAR2 $\mathrm{ASV}^{+}$patients, who had more severe peritumoral brain edema, tumor invasion in more brain lobes and a shorter median survival time compared with ADAR2 ASVpatients. Therefore, it appears that ADAR2 mRNA levels are not altered in glioma-derived cell lines or glioma tissues, the self-editing of the ADAR2 pre-mRNA generates an ADAR2 ASV in glioma-derived cell lines and glioma tissues, and the expression of this ADAR2 ASV may be associated with the malignancy of glioma.

Early studies by the present authors lead to a hypothesis that ADAR2 splicing isoforms may affect its enzyme activity; therefore leading to investigations concerning the association between splicing isoform expression and the clinical features of glioma. However, the identified percentage of ADAR2 ASVs (10-25\%) was not enough to theoretically explain the reduced RNA editing of the GluA2 Q/R site. Hideyama and Kwak (56) demonstrated that expression of $\mathrm{Q} / \mathrm{R}$ site-unedited GluA2 requires $>50 \%$ reduction of ADAR 2 activity; therefore, the present authors analyzed the differences in the alternative splicing patterns of ADAR2 in glioma U87, U251 and A172 cell lines and normal human astrocytes HA1800 cells (50). Quantitative polymerase chain reaction identified no significant differences in the ADAR2 pre-mRNA splicing patterns at exon 1a or 2 between the glioma-derived cell lines and normal human astrocytes. However, transcripts including exon 5a were predominantly expressed in the glioma-derived cell lines, and transcripts without exon 5a were relative to its expression in normal human astrocytes. Taken together, these findings indicate that alternative splicing in glioma cells causes an abnormal increase in the expression of exon 5a, leading to the active suppression of ADAR2 activity and a reduction in A-to-I RNA editing.

Therefore, A-to-I RNA editing is regulated by the pattern of ADAR2 alternative splicing in glioma. Collectively, in vitro results suggest an association between the increased expression of abnormal ADAR2 isoforms or specific ADAR2 ASVs and the malignant characteristics of astrocytoma. Further studies concerning human glioma tissues are required to support in vitro findings, using normal brain white matter as a control. The primary aim of such a study would be to determine whether the alterations in ADAR2 ASV expression may be used as a novel marker for the molecular classification of glioma and to monitor tumor progression in patients.

\section{Conclusion}

In summary, abnormal expression and activity of ADAR2 contributes to abnormal RNA editing. Additional study into specific ADAR2 ASVs is required to identify the specific RNA abnormalities that are associated with tumorigenesis and tumor development, and to determine the complex associations between ADAR-mediated A-to-I editing and the alternative splicing of pre-mRNA ADAR2 in specific diseases. Furthermore, additional study is required to establish the precise regulation of A-to-I editing by ADAR2 ASVs and how they contribute to glioma genesis and progression. Overall, future aims would be to identify novel ADAR2 target genes, investigate the rescue potential of ADAR2 editing activity and define the underlying rules of ADAR2 ASVs differential expression. Identification of subtle alterations in the transcriptome introduced by A-to-I RNA editing and ADAR2 isoforms with differential activity generated by alternative splicing may continue to lead to additional and notable findings.

\section{Acknowledgements}

The authors wish to acknowledge the funding agencies that supported certain original work cited in this review: The National Science Foundation of China (Beijing, China; grant no. 30672159) and the Doctoral Program of Higher Education Research Fund (Beijing, China; grant no. 20110061110070).

\section{References}

1. Maas S, Patt S, Schrey M and Rich A: Underediting of glutamate receptor GluR-B mRNA in malignant gliomas. Proc Natl Acad Sci USA 98: 14687-14692, 2001.

2. Cenci C, Barzotti R, Galeano F, Corbelli S, Rota R, Massimi L, Di Rocco C, O'Connell MA and Gallo A: Down-regulation of RNA editing in pediatric astrocytomas: ADAR2 editing activity inhibits cell migration and proliferation. J Biol Chem 283: 7251-7260, 2008.

3. Li Z, Tian Y, Tian N, Zhao X, Du C, Han L and Zhang H: Aberrant alternative splicing pattern of ADAR2 downregulates adenosine-to-inosine editing in glioma. Oncol Rep 33: 2845-2852, 2015.

4. Benne R, Van Den Burg J, Brakenhoff JP, Sloof P, Van Boom JH and Tromp MC: Major transcript of the frameshifted coxll gene from trypanosome mitochondria contains four nucleotides that are not encoded in the DNA. Cell 46: 819-826, 1986.

5. Rebagliati MR and Melton DA: Antisense RNA injections in fertilized frog eggs reveal an RNA duplex unwinding activity. Cell 48: 599-605, 1987.

6. Wagner RW, Smith JE, Cooperman BS and Nishikura K: A double-stranded RNA unwinding activity introduces structural alterations by means of adenosine to inosine conversions in mammalian cells and Xenopus eggs. Proc Natl Acad Sci USA 86: 2647-2651, 1989.

7. Tang W, Fei Y and Page M: Biological significance of RNA editing in cells. Mol Biotechnol 52: 91-100, 2012.

8. Osato D, Rogers K, Guo Q, Li F, Richmond G, Klug F and Simpson L: Uridine insertion/deletion RNA editing in trypanosomatid mitochondria: In search of the editosome. RNA 15: 1338-1344, 2009.

9. Visomirski-Robic LM and Gott JM: Insertional editing in isolated Physarum mitochondria is linked to RNA synthesis. RNA 3: 821-837, 1997.

10. Doe CM, Relkovic D, Garfield AS, Dalley JW, Theobald DE, Humby T, Wilkinson LS and Isles AR: Loss of the imprinted snoRNA mbii-52 leads to increased 5 htr $2 \mathrm{c}$ pre-RNA editing and altered 5HT2CR-mediated behaviour. Hum Mol Genet 18: 2140-2148, 2009. 
11. Klipcan L, Moor N, Kessler N and Safro MG: Eukaryotic cytosolic and mitochondrial phenylalanyl-tRNA synthetases catalyze the charging of tRNA with the meta-tyrosine. Proc Natl Acad Sci USA 106: 11045-11048, 2009.

12. Lynch M, Koskella B and Schaack S: Mutation pressure and the evolution of organelle genomic architecture. Science 311: 1727-1730, 2006.

13. Sommer B, Kohler M, Sprengel R and Seeburg PH: RNA editing in brain controls a determinant of ion flow in glutamate-gated channels. Cell 67: 11-19, 1991.

14. Nishikura K: Functions and regulation of RNA editing by ADAR deaminases. Annu Rev Biochem 79: 321-349, 2010.

15. Bazak L, Haviv A, Barak M, Jacob-Hirsch J, Deng P, Zhang R, Isaacs FJ, Rechavi G, Li JB, Eisenberg E and Levanon EY: A-to-I RNA editing occurs at over a hundred million genomic sites, located in a majority of human genes. Genome Res 24: 365-376, 2014.

16. Macbeth MR, Schubert HL, Vandemark AP, Lingam AT, Hill CP and Bass BL: Inositol hexakisphosphate is bound in the ADAR2 core and required for RNA editing. Science 309: 1534-1539, 2005.

17. Ryter JM and Schultz SC: Molecular basis of double-stranded RNA-protein interactions: Structure of a dsRNA-binding domain complexed with dsRNA. EMBO J 17: 7505-7513, 1998.

18. Kuttan A and Bass BL: Mechanistic insights into editing-site specificity of ADARs. Proc Natl Acad Sci USA 109: E3295-E3304, 2012.

19. Slotkin W and Nishikura K: Adenosine-to-inosine RNA editing and human disease. Genome Med 5: 105, 2013.

20. Chen CX, Cho DS, Wang Q, Lai F, Carter KC and Nishikura K: A third member of the RNA-specific adenosine deaminase gene family, ADAR3, contains both single- and double-stranded RNA binding domains. RNA 6: 755-767, 2000.

21. Levanon EY, Eisenberg E, Yelin R, Nemzer S, Hallegger M, Shemesh R, Fligelman ZY, Shoshan A, Pollock SR, Sztybel D, et al: Systematic identification of abundant A-to-I editing sites in the human transcriptome. Nat Biotechnol 22: 1001-1005, 2004.

22. Collingridge GL, Olsen RW, Peters J and Spedding M: A nomenclature for ligand-gated ion channels. Neuropharmacology 56: $2-5,2009$.

23. Higuchi MSF, Köhler M, Sommer B, Sprengel R and Seeburg PH: RNA editing of AMPA receptor subunit GluR-B: A basepaired intron-exon structure determines position and efficiency. Cell 75 : 1361-1370, 1993.

24. Barresi S, Tomaselli S, Athanasiadis A, Galeano F, Locatelli F, Bertini E, Zanni G and Gallo A: Oligophrenin-1 (OPHN1), a gene involved in X-linked intellectual disability, undergoes RNA editing and alternative splicing during human brain development. PLoS One 9: e91351, 2014

25. Hogg M, Paro S, Keegan LP and O'Connell MA: RNA editing by mammalian ADARs. Adv Genet 73: 87-120, 2011.

26. Yang W, Chendrimada TP, Wang Q, Higuchi M, Seeburg PH, Shiekhattar R and Nishikura K: Modulation of microRNA processing and expression through RNA editing by ADAR deaminases. Nat Struct Mol Biol 13: 13-21, 2006.

27. Vesely C, Tauber S, Sedlazeck FJ, Tajaddod M, Haeseler AV and Jantsch MF: ADAR2 induces reproducible changes in sequence and abundance of mature microRNAs in the mouse brain. Nucleic Acids Res 42: 12155-12168, 2014.

28. Yang Y, Zhou X and Jin Y: ADAR-mediated RNA editing in non-coding RNA sequences. Sci China Life Sci 56: 944-952, 2013.

29. Hundley HA and Bass BL: ADAR editing in double-stranded UTRs and other noncoding RNA sequences. Trends Biochem Sci 35: 377-383, 2010

30. Berget SM, Moore C and Sharp PA: Spliced segments at the 5 terminus of adenovirus 2 late mRNA. Proc Natl Acad Sci USA 74: 3171-3175, 1977.

31. Sambrook J: Adenovirus amazes at Cold Spring Harbor. Nature 268: 101-104, 1977.

32. Gilbert W: Why genes in pieces? Nature 271: 501, 1978.

33. Brett D, Pospisil H, Valcarcel J, Reich J and Bork P: Alternative splicing and genome complexity. Nat Genet 30: 29-30, 2002.

34. Sharp PA: Split genes and RNA splicing. Cell 77: 805-815, 1994
35. Lander ES, Linton LM, Birren B, Nusbaum C, Zody MC, Baldwin J, Devon K, Dewar K, Doyle M, FitzHugh W, et al: Initial sequencing and analysis of the human genome. Nature 409: 860-921, 2001

36. Sharp PA: The discovery of split genes and RNA splicing. Trends Biochem Sci 30: 279-281, 2005.

37. Wang ET, Sandberg R, Luo S, Khrebtukova I, Zhang L, Mayr C, Kingsmore SF, Schroth GP and Burge CB: Alternative isoform regulation in human tissue transcriptomes.Nature 456: 470-476, 2008.

38. Pan Q, Shai O, Lee LJ, Frey BJ and Blencowe BJ: Deep surveying of alternative splicing complexity in the human transcriptome by high-throughput sequencing. Nat Genet 40: 1413-1415, 2008.

39. Venables JP: Aberrant and alternative splicing in cancer. Cancer Res 64: 7647-7654, 2004.

40. Tomaselli S, Bonamassa B, Alisi A, Nobili V, Locatelli F and Gallo A: ADAR enzyme and miRNA story: A nucleotide that can make the difference. Int J Mol Sci 14: 22796-22816, 2013.

41. Mittaz L, Scott HS, Rossier C, Seeburg PH, Higuchi M and Antonarakis SE: Cloning of a human RNA editing deaminase (ADARB1) of glutamate receptors that maps to chromosome 21q22.3. Genomics 41: 210-217, 1997.

42. Slavov D and Gardiner K: Phylogenetic comparison of the pre-mRNA adenosine deaminase ADAR2 genes and transcripts: Conservation and diversity in editing site sequence and alternative splicing patterns. Gene 299: 83-94, 2002.

43. Kawahara Y, Ito K, Ito M, Tsuji S and Kwak S: Novel splice variants of human ADAR2 mRNA: Skipping of the exon encoding the dsRNA-binding domains and multiple C-terminal splice sites. Gene 363: 193-201, 2005.

44. Gerber A, O'Connell MA and Keller W: Two forms of human double-stranded RNA-specific editase 1 (hRED1) generated by the insertion of an Alu cassette. RNA 3: 453-463, 1997.

45. Lai F, Chen CX, Carter KC and Nishikura K: Editing of glutamate receptor B subunit ion channel RNAs by four alternatively spliced DRADA2 double-stranded RNA adenosine deaminases. Mol Cell Biol 17: 2413-2424, 1997.

46. Rueter SM, Dawson TR and Emeson RB: Regulation of alternative splicing by RNA editing. Nature 399: 75-80, 1999.

47. Maas $S$ and Gommans WM: Novel exon of mammalian ADAR2 extends open reading frame. PLoS One 4: e4225, 2009.

48. Agranat L, Sperling J and Sperling R: A novel tissue-specific alternatively spliced form of the A-to-I RNA editing enzyme ADAR2. RNA Biol 7: 253-262, 2010.

49. Solomon O, Oren S, Safran M, Deshet-Unger N, Akiva P, Jacob-Hirsch J, Cesarkas K, Kabesa R, Amariglio N, Unger $\mathrm{R}$, et al: Global regulation of alternative splicing by adenosine deaminase acting on RNA (ADAR). RNA 19: 591-604, 2013.

50. Galeano F, Tomaselli S, Locatelli F and Gallo A: A-to-I RNA editing: The 'ADAR' side of human cancer. Semin Cell Dev Biol 23: 244-250, 2012.

51. Dominissini D, Moshitch-Moshkovitz S, Amariglio N and Rechavi G: Adenosine-to-inosine RNA editing meets cancer. Carcinogenesis 32: 1569-1577, 2011.

52. Tomaselli S, Galeano F, Massimi L, Di Rocco C, Lauriola L, Mastronuzzi A, Locatelli F and Gallo A: ADAR2 editing activity in newly diagnosed versus relapsed pediatric high-grade astrocytomas. BMC Cancer 13: 255, 2013.

53. Choudhury Y, Tay FC, Lam DH, Sandanaraj E, Tang C, Ang BT and Wang S: Attenuated adenosine-to-inosine editing of microRNA-376a* promotes invasiveness of glioblastoma cells. J Clin Invest 122: 4059-4076, 2012.

54. Galeano F, Rossetti C, Tomaselli S, Cifaldi L, Lezzerini M, Pezzullo M, Boldrini R, Massimi L, Di Rocco CM, Locatelli F and Gallo A: ADAR2-editing activity inhibits glioblastoma growth through the modulation of the CDC14B/Skp2/p21/p27 axis. Oncogene 32: 998-1009, 2013.

55. Wei J, Li ZH, Du C, Qi B, Zhao X, Wang L, Bi L, Wang G, Zhang X, Su X, et al: Abnormal expression of an ADAR2 alternative splicing variant in gliomas downregulates adenosine-to-inosine RNA editing. Acta Neurochir (Wien) 156: 1135-1142, 2014.

56. Hideyama T and Kwak S: When Does ALS Start? ADAR2-GluA2 Hypothesis for the Etiology of Sporadic ALS. Front Mol Neurosci 4: 33, 2011 